\title{
Atomic Resolution STEM-EELS Studies of Defects and Local Structural Distortions in Oxide Interfaces
}

\author{
G. Sánchez-Santolino ${ }^{1}$, M. A. Roldan ${ }^{2}$, Qiao Qiao ${ }^{3}$, L. Begon-Lours ${ }^{4}$, M. A. Frechero ${ }^{2}$, J. Salafranca ${ }^{2}$, R. \\ Mishra $^{5}$, C. Leon ${ }^{2}$, S. T. Pantelides ${ }^{6}$, S. J. Pennycook ${ }^{7}$, J. E. Villegas ${ }^{4}$ J. Santamaria ${ }^{2}$ and M. Varela ${ }^{2}$ \\ 1. Crystal Interface Laboratory, University of Tokyo, Tokyo 113-8656, Japan. \\ 2. GFMC, Dept. de Fisica de Materiales, Universidad Complutense de Madrid, 28040 Madrid, Spain. \\ ${ }^{3}$ Temple University, Philadelphia PA 19122 \& Brookhaven National Laboratory, Upton NY 11973. \\ 4. Unité Mixte de Physique CNRS/Thales, Palaiseau 91767 (France). \\ 5. Dept. of Mechanical Engineering and Materials Science, Washington University in St. Louis, St. \\ Louis, MO 63130 \\ ${ }^{6 .}$ Dept. of Physics and Astronomy, Vanderbilt University, Nashville, TN 37235, USA \\ 7. Dept. of Materials Science \& Engineering, National University of Singapore, Singapore 117575.
}

Doped complex oxides show a wide range of interesting properties due to a strong interplay and competition between lattice, spin, and charge degrees of freedom. In these systems, subtle changes in local structure or chemistry may result in colossal responses in macroscopic physical behavior. In this talk we will apply atomic resolution aberration corrected scanning transmission electron microscopy (STEM) and electron energy-loss spectroscopy (EELS) to the study of the chemistry and local structure around defects, near complex oxide interfaces, and grain boundaries. Thanks to spherical aberration correction, both spatial resolution and sensitivity limits attainable in the STEM have improved down to the single-atom level, resulting in unprecedented contrast and signal-to-noise ratio improvements in both imaging and EELS. We will discuss a few examples where atomic resolution compositional mapping constitutes a key task to understand the system physical properties, highlighting the importance of considering artifacts during quantification.

The first example will include a study of ferroelectric/ superconducting/ insulating $\mathrm{BiFeO}_{3}(\mathrm{BFO})$ / $\mathrm{YBa}_{2} \mathrm{Cu}_{3} \mathrm{O}_{7}$ (YBCO) / $\mathrm{PrBa}_{2} \mathrm{Cu}_{3} \mathrm{O}_{7}$ (PBCO) heterostructures grown on $\mathrm{SrTiO}_{3}$ (STO) (001) substrates. Ferroelectricity in BFO can be used to modify the local doping of the superconductor polarization through externally applied electric fields. Asymmetric doping effects are found when the field is reversed. Atomic resolution high angle annular dark field (HAADF) images combined with EELS maps show that the $\mathrm{BFO}$ interfaces are $\mathrm{FeO}_{2}$ terminated. This finding, combined with the fact that the polarization of as-grown BFO layers points in a preferential direction (Figure 1), modifies the YBCO hole doping near the interface. These effects can be understood considering the interplay between interface polarity and charge transfer phenomena.

Another example where local strains and defects rule the macroscopic properties can be found in ionic conductors such as those in solid electrolytes. Their physical properties are often limited by ion blocking at grain boundaries so understanding the chemical, structural and electronic properties of these materials at the atomic scale is the key to improve functional properties. Atomic resolution STEM-EELS of single grain boundaries in bicrystals of yttria $(9 \% \mathrm{~mol})$ stabilized zirconia (YSZ) reveal a strong yttrium segregation at the expansive site of the grain boundary cores (see Figure 2), doubling the bulk relative Y concentration [1]. Such chemical segregation at dislocation cores results in the generation of static $\mathrm{O}$ vacancies that affect the local electrostatic potential and hence, the macroscopic ionic conduction properties. We will discuss these findings in the light of density-functional theory calculations. 
References:

[1] M. A. Frechero et al. Scientific Reports, 5 (2015) 17229.

[2] Research at UCM sponsored by Spanish MICINN through grants MAT2008-06517-C02 and

Consolider Ingenio 2010 - CSD2009-00013 (Imagine), by CAM through grant S2009/MAT-1756

(Phama), Spanish MINECO/FEDER MAT2015-66888-C3-3-R and by the ERC Proof of Concept Grant MAGTOOLS. Research at ORNL was sponsored by the Materials Sciences and Engineering Division of the U.S. Department of Energy (DOE) and ORNL's Center for Nanophase Materials User Program.
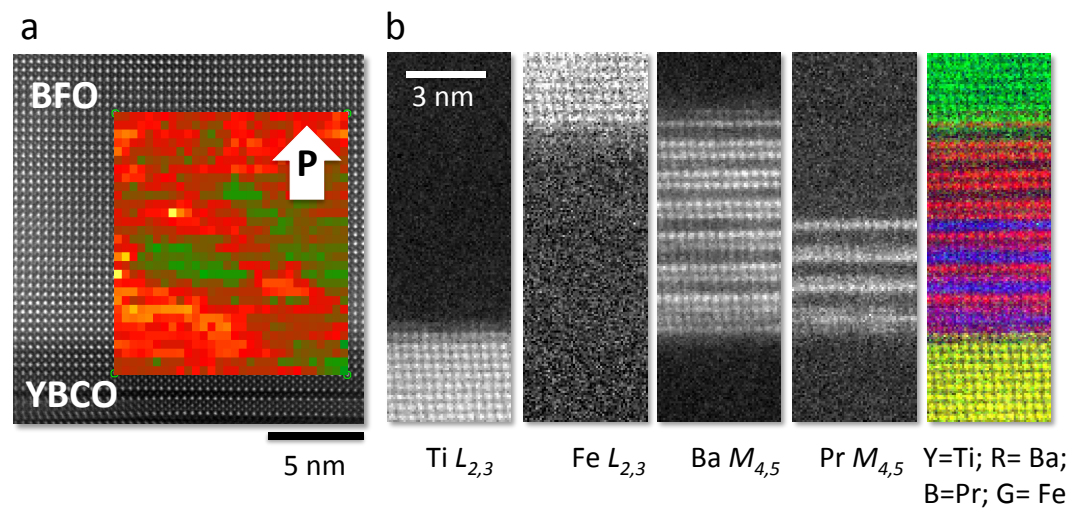

Figure 1. (a) Atomic resolution HAADF image of a BFO/YBCO interface. The overlaid map exhibits a map of the local distortions (in false colour, on a matching scale), which correlate with the local ferroelectric polarization, P. (b) EELS maps obtained across a BFO (top) / YBCO (middle) / PBCO (bottom) heterostructure. Some structural defects and interface steps are observed. From left to right: atomic resolution, normalized integrated signal maps of the $\operatorname{Ti} L_{2,3}$, $\operatorname{Fe} L_{2,3}$, $\mathrm{Ba} M_{4,5}$ and $\operatorname{Pr} M_{4,5}$ edges, respectively. The right panel exhibits a color map where all chemical images have been overlaid.
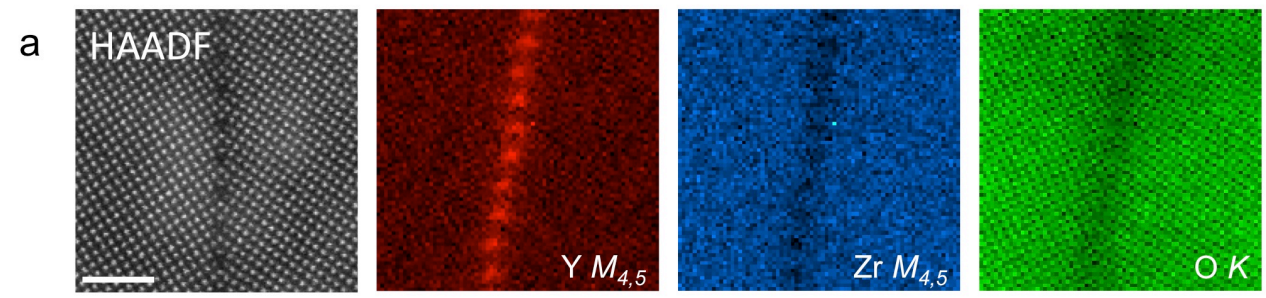

b
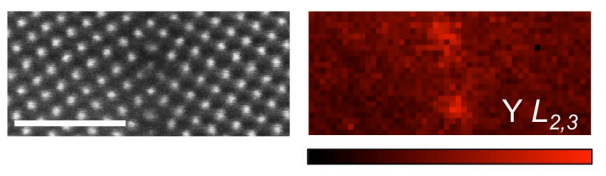

$0 \%$

$18 \%$
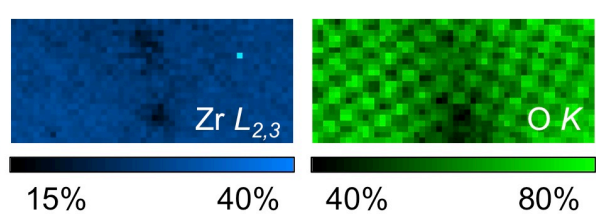

Figure 2. EELS maps of a YSZ grain boundary. (a) Spectrum image of the grain boundary region obtained in a Nion UltraSTEM100 operated at 100kV. From left to right: Z-contrast image of the grain boundary; atomic resolution, normalized integrated signal maps of the $\mathrm{Y} M_{4,5}$ (red), $\mathrm{Zr} M_{4,5}$ (blue) and $\mathrm{O}$ $K$ (green) edges. (b) From left to right: Z-contrast image of the grain boundary; atomic resolution, normalized integrated signal maps of $\mathrm{Y}_{2,3}$ (red), $\mathrm{Zr} L_{2,3}$ (blue) and $\mathrm{O} K$ (green) edges, respectively. Scale bars represent $2 \mathrm{~nm}$. Data obtained in a Nion UltraSTEM200 operated at 200kV. Adapted from reference [1]. 